\title{
Im Vierklang
}

\section{Erhard Taverna}

erhard.taverna@saez.ch
In der Reihenfolge ihres Eintreffens sind vier Bücher zu besprechen: «Sportverletzt - was jetzt?» [1], «Samenspende» [2], «Intelligente Emotionalität» [3] und «Wer nicht denken will, muss laufen» [4]. Ein hervorragendes Sachbuch aus Deutschland, ein spannender Kriminalroman aus ärztlicher Hand, ein informatives Werk über das Universum der Gefühle und ein wohltuend ironisches Geschichtenbuch aus der Anästhesie. Emotionen sind immer im Spiel, beim Wettkampf, beim Morden, beim Analytiker, weniger bei Narkotisierten, dafür umso mehr beim Personal.

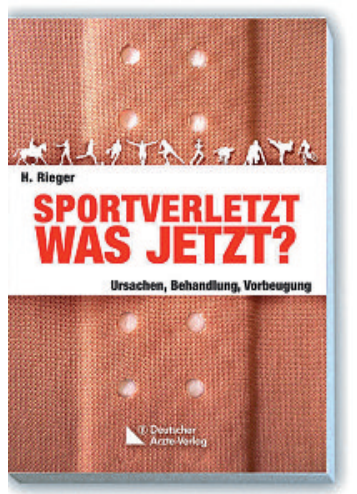

Horst Rieger. Sportverletzt. Was jetzt? Köln: Deutscher Ärzteverlag; 2009. 817 Seiten. Fr. 80.90

ISBN 978-3-7691-0603-9.

\section{Sportverletzt - was jetzt?}

Aus Deutschland, genauer aus dem Clemensspital in Münster, kommt das Fachbuch über die weniger erfreulichen Seiten des Sports, über Verletzungsursachen, Behandlung und Vorbeugung. Klar formuliert und reich bebildert, richtet sich der Autor und Chefarzt laut Vorwort an Sportlerinnen und Sportler, Trainer, Sportlehrer, Betreuer und Manager, Physiotherapeuten, Mediziner, Sport- und Medizinstudenten, Sportjournalisten sowie Sportbegeisterte, die nicht mehr aktiv sein können. Der Orthopäde schreibt nicht für die Spezialistenkollegen, aber sehr wohl für traumatologisch tätige Allgemeinärzte. Sie finden darin eine praxisorientierte Anleitung für das ganze Spektrum akuter und chronischer Leiden des Bewegungsapparates aller Altersstufen. Vor allem das schwergewichtige 2. Kapitel behandelt in ausgezeichneter Form Diagnosen und Therapien der Sportverletzungen und Überlastungsschäden von Kopf bis Fuss. Konservative und operative Indikationen sind klar voneinander abgegrenzt, ihre Vorund Nachteile benannt, «banale» und «komplizierte» Verletzungen werden mit der gleichen Sorgfalt abgehandelt.

Die Aufmerksamkeit des erfahrenen Orthopäden gilt den relevanten und häufigen Problemen, wie sie in jeder breitgefächerten Praxis vorkommen: den Nagel- verletzungen, Muskelprellungen und Gelenkproblemen von Chassaignac bis Perthes, den Ansatztendinosen, Impingementsyndromen und komplexeren Knochenbrüchen. Abklärungsmethoden sind vorgestellt, Komplikationen beschrieben, alternative Therapiemöglichkeiten abgewogen. Richtigerweise werden die besonders häufigen Probleme an den Händen, Sprunggelenken und Füssen ausführlich abgehandelt. Wer Frakturen, wie Radius-, Tibia- oder subcapitale Humerusfrakturen und andere, in eigener Regie behandelt, wird auf detailliertere Informationen zugreifen. Das Buch garantiert aber eine breite, fundierte Übersicht, versehen mit einem gut brauchbaren Stichwortverzeichnis.

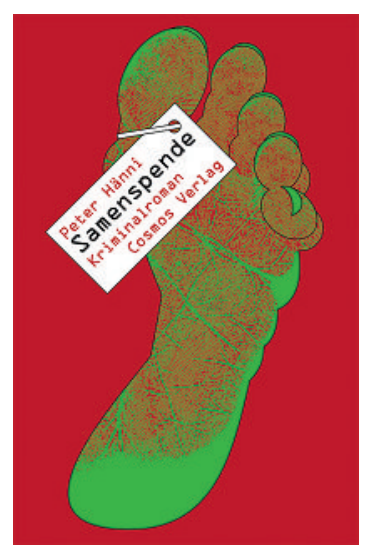

Peter Hänni. Samenspende

Muri b. Bern: Cosmos; 2009. 189 Seiten.

Fr. 34.-.

ISBN 978-3-305-00430-0.

\section{Samenspende}

In Berns Parkanlagen wächst auch die Christuspalme. Wer daraus noch anderes als Rizinusöl gewinnen möchte, lernt hier die Pflanze gründlicher kennen. Ältere erinnern sich vielleicht noch an die Ermordung eines bulgarischen Schriftstellers im London der 70er Jahre, bei der ein Regenschirm eine verhängnisvolle Rolle spielte. Von den toxikologischen Zusammenhängen erfahren Leserinnen und Leser auf einem Parcours durch die Notfallstation des Inselspitals, den überbelegten Seziersaal der Medizinklinik und die Labors der Gerichtsmedizin. Schicksalhaft geht alles auf eine lange zurückliegende Samenspende zurück, von der ordnungsgemäss nur das Eidgenössische Amt für das Zivilstandswesen Genaueres weiss. Der Autor versteht es erneut, uns informativ und listenreich in ein gefährliches Labyrinth zu locken. Gute medizinische Kenntnisse sind gefragt und viel polizeiliche Ermittlung. Ein Kommissar mit anhänglichem Hund, ein lädierter Mitarbeiter und sein Kollege treiben die Handlung zügig voran. Das Ende bringt auch nicht gerade das, was wir von einem Kuss erwarten. 


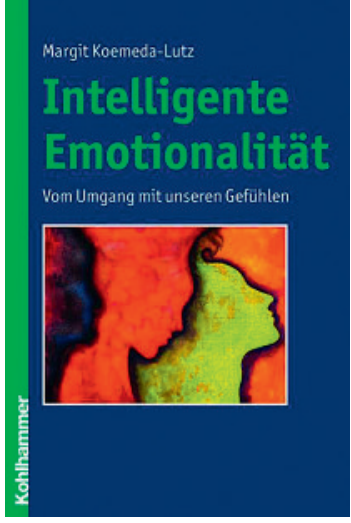

Margit Koemeda-Lutz. Intelligente Emotionalität. Vom Umgang mit unseren Gefühlen

Stuttgart: Kohlhammer; 2009. 153 Seiten.

Fr. 39.90 .

ISBN 978-3-17-020839-1

\section{Intelligente Emotionalität}

Vom Umgang mit unseren Gefühlen handelt das Buch der bioenergetisch orientierten Psychologin. Emotionales Lernen ist vor allem soziales Lernen. Wahrnehmung, Empfindung, Erinnerung, Denken und Handeln sind untrennbar mit Gefühlen verknüpft. Die Autorin schildert in einem ersten Teil gut verständlich den Stand der neurobiologischen Forschung, insbesondere die Strukturen des limbischen Systems, die Rolle der Neuromodulatoren, Neuropeptide und Neurohormone. Ein zweiter Teil ist den Anwendungen in der psychotherapeutischen Praxis gewidmet, wo an Beispielen krankmachender Emotionen wie Trauer, Depression, Ärger, Angst oder Wut Therapiemöglichkeiten vorgestellt werden. Die emotionale Alltagspraxis

«Was sich im wirklichen Leben abspielt, stellt «Emergency Room» weit in den Schatten»

gleicht einer Gratwanderung. Weil Stimmungen und Gefühle stärker handlungsleitend sind als Gedanken, erfordern Kommunikation und soziale Wirkung eine emotionale Kontrolle. Wo sollen wir Gefühle zulassen, wo zügeln? Die Autorin möchte uns einladen, «die Energie unserer Gefühle genauer zu untersuchen, den Umgang damit zu kultivieren, um die Entfaltung destruktiver Wirkungen möglichst zu minimieren und ihre belebenden und wohltuenden Aspekte nutzen zu lernen». Gut reguliert und intelligent sollen wir damit umgehen, auf dass Emotionen uns «dahin führen, wo wir unmittelbar betroffen sind - zum Anwesendsein mit Haut und Haar, zur Fülle unserer Lebendigkeit».

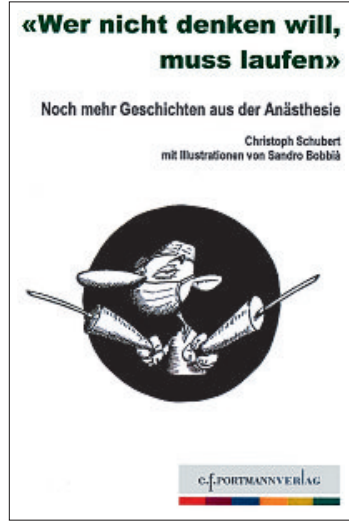

Christoph Schubert. Wer nicht denken will muss laufen.

Noch mehr Geschichten aus der Anästhesie.

Mit Illustrationen von Sandro Bobbià. Portmann; 2009.

\section{«Wer nicht denken will, muss laufen»}

Der liebe Gott möge dem beistehen, der einem der unzähligen operativ tätigen Heiler in die Finger gerät. Weil jener das leider nicht kann, hat er diese Aufgabe an seine Schutzengel delegiert. Wie das leicht vorauszusehen ist, besorgen das stellvertretend die Anästhesisten, die verborgen in der Unterwelt der Operationssäle und Intensivstationen ihr gutes Werk verrichten. Schon einmal hat Christoph Schubert von diesen mehr oder weniger stillen Helden erzählt. Jetzt winkt er uns noch einmal als Schutzengel freundlich zu und vertieft seine Aufklärungsarbeit mit weiteren Reportagen aus der Arbeitswelt, prosaisch und gereimt, humorvoll und deftig. Was sich im wirklichen Leben abspielt, stellt «Emergency Room» weit in den Schatten. Zudem ist das Einfühlungsvermögen des Autors fast grenzenlos. Selten noch hat ein Darmbewohner namens Anton so eindringlich seine abenteuerliche Reise zum Sphinkter beschrieben, und was uns ein Garderobenschrank für die Herren der OP-Abteilung berichtet, leuchtet tief in medizinische Abgründe. Sparmassnahmen werden auf die Schippe genommen, schwierige Patienten und noch schwierigere Kollegen, die gnadenlose Hackordnung der Klinik und ein finales Spitalfest mit Blick in die Zukunft. Der Schreiber weiss als leidender Facharzt, wovon er redet, sonst hätte er nicht einen Kinderarzt als begabten Cartoonist zur Seite. Aller guten Dinge sind zwei, ausser bei Pistazieneiskugeln, und einen dritten Band werde es nicht mehr geben, verrät uns das Duo am Schluss. Vielleicht können da die Leserinnen und Leser noch mitentscheiden. 